\title{
A Collocation-Galerkin Method for Poisson's Equation on Rectangular Regions
}

\author{
By Julio César Díaz
}

\begin{abstract}
A collocation-Galerkin method is defined for Poisson's equation on the unit square, using tensor products of continuous piecewise polynomials. Optimal $L^{2}$ and $H_{0}^{1}$ orders of convergence are established. This procedure requires fewer quadratures than the corresponding Galerkin procedure.
\end{abstract}

Introduction. The collocation-Galerkin method was first introduced by Diaz [2], [3] for the two-point boundary value problems and optimal $L^{2}$-rates of convergence were established for a particular choice of the collocation points; namely, the affine images of the roots of a Jacobi polynomial. In [6] Wheeler derived optimal $L^{p}$-estimates and applied this method to a one space dimensional parabolic problem. In [4] Dunn and Wheeler analyzed some collocation- $H^{-1}$-Galerkin methods and established optimal $L^{p}$. estimates for any choice of the collocation points. Archer and Diaz [1] have applied similar ideas to a one-dimensional first order hyperbolic problem and derived optimal $L^{2}$-estimates. Here, a collocation-Galerkin method is defined for Poisson's equation on the unit square, using tensor products of continuous piecewise polynomials and the collocation points are based on the roots of a Jacobi polynomial. Optimal $L^{2}$ - and $H_{0}^{1}$-estimates are established. On the basis of computational complexity the collocation-Galerkin method is intermediate between the Galerkin method and the collocation method. It has an advantage over the Galerkin procedure for the same space in that the integrals involve the product of the approximate solution and a piecewise linear function, thus the integrals are simpler and, of course, there are fewer of them. Also, the continuity conditions on the approximate solution are weaker than those required of the collocation approximation defined by Prenter and Russell [5].

In the following section, the collocation-Galerkin method is defined and existence and uniqueness are shown using some semidiscrete bilinear forms. In the last section, the error analysis is presented. The analysis consists of reducing the problem to some one-dimensional problems for which the results of [3] can be applied.

The Collocation-Galerkin Method. Consider the boundary value problem

$$
\begin{aligned}
-\Delta u=f, & \text { on } \Omega, \\
u=0, & \text { on } \partial \Omega,
\end{aligned}
$$

where $\Omega=(0,1) \times(0,1)$. We shall assume that there exists a unique $u$ and that it sufficiently smooth.

Received March 8, 1976 ; revised March 24, 1978.

AMS (MOS) subject classifications (1970). Primary 65N35, 65N30. 
For a partition $\delta=\left\{x_{i}\right\}_{i=1}^{N+1}$ of the unit interval $I=[0,1]$ satisfying,

let

$$
0=x_{0}<x_{1}<\cdots<x_{N+1}=1 \text {, }
$$

$$
\begin{aligned}
& y_{l}=x_{l}, \quad l=0, \ldots, N+1, \\
& I_{i}=\left[x_{i}, x_{i+1}\right], \quad h_{i}=x_{i+1}-x_{i}, \quad i=0, \ldots, N, \\
& h=\max _{0 \leqslant i \leqslant N} h_{i} .
\end{aligned}
$$

If $r \geqslant 1$ is an integer, let $\mathbf{P}_{r}(E)$ denote the class of polynomials of degree at most $r$ on the set $E$, and define

$$
M_{0}^{r} \equiv M_{0}^{r}(\delta)=\left\{V \in C(I) \mid V \in \mathrm{P}_{r}\left(I_{i}\right), i=0, \ldots, N, V(0)=V(1)=0\right\} .
$$

Let $\varphi_{1}, \ldots, \varphi_{r-1}$ and positive $\omega_{1}, \ldots, \omega_{r-1}$ be the unique choices such that

$$
\int_{0}^{1} x(1-x) p(x) d x=\sum_{j=1}^{r-1} \omega_{j} p\left(\varphi_{j}\right), \quad \forall p \in \mathbf{P}_{2 r-3}(0,1) .
$$

That is, $\varphi_{1}, \ldots, \varphi_{r-1}$ are the roots of the Jacobi polynomial $J_{r-1}$ of degree $r-1$ on $[0,1]$ with respect to the weight function $x(1-x)$. The collocation points are tensor products of affine transformations of the roots of $J_{r-1}$ onto each subinterval. More precisely, let $x_{i j}=x_{i}+h_{i} \varphi_{j}, y_{l k}=y_{l}+h_{l} \varphi_{k}, i, l=0, \ldots, N ; j, k=1, \ldots, r-1$.

The collocation-Galerkin method for the approximate solution of (1) consists in finding $U \in M=M_{0}^{r} \otimes M_{0}^{r}$ satisfying

$$
\Delta U\left(x_{i j}, x_{l k}\right)+f\left(x_{i j}, x_{l k}\right)=0, \quad \begin{aligned}
& i, l=0, \ldots, N, \\
& j, k=1, \ldots, r-1,
\end{aligned}
$$

$$
\begin{gathered}
\int_{I} \frac{\partial^{2}}{\partial x^{2}}\left(U\left(x_{i j}, \xi\right)\right) V(\xi) d \xi-\int_{I} \frac{\partial}{\partial y}\left(U\left(x_{i j}, \xi\right)\right) V^{\prime}(\xi) d \xi \\
+\int_{I} f\left(x_{i j}, \xi\right) V(\xi) d \xi=0, \quad \begin{array}{l}
i=0, \ldots, N, \quad j=1, \ldots, r-1, \\
j=
\end{array}, \forall V \in M_{0}^{1}, \\
-\int_{I} \frac{\partial}{\partial x}\left(U\left(\xi, y_{l k}\right)\right) V^{\prime}(\xi) d \xi+\int_{I} \frac{\partial^{2}}{\partial y^{2}}\left(U\left(\xi, y_{l k}\right)\right) V(\xi) d \xi \\
+\int_{I} f\left(\xi, y_{l k}\right) V(\xi) d \xi=0, \quad l=0, \ldots, N, \quad k=1, \ldots, r-1, \quad \forall V \in M_{0}^{1}, \\
(\nabla U, \nabla V)=(f, V), \quad \forall V \in M_{0}^{1} \otimes M_{0}^{1},
\end{gathered}
$$

where $(\cdot, \cdot)$ denotes the $L^{2}$-inner product over $\Omega$.

In order to demonstrate the existence and uniqueness of the collocation-Galerkin approximation $U$, bilinear forms $D(\cdot, \cdot), D$ and $I$ are introduced. Let

Then

$$
Z_{0}^{r}=\left\{V \in M_{0}^{r} \mid V\left(x_{i}\right)=0, i=1, \ldots, N\right\} .
$$

$$
M_{0}^{r}=M_{0}^{1} \oplus Z_{0}^{r}
$$


For $v \in Z_{0}^{r}$ and a function $g$ defined on $I$, let

and set

$$
D_{i}(g, V)=\sum_{j=1}^{r-1} h_{i} \omega_{j} \frac{g\left(x_{i j}\right) V\left(x_{i j}\right)}{\varphi_{j}\left(1-\varphi_{j}\right)},
$$

$$
D(g, V)=\sum_{i=0}^{N} D_{i}(g, V) .
$$

Two semidiscrete bilinear forms $D(\cdot, \cdot)$ and $I(\cdot, \cdot)$ are defined as follows; for $V \in M_{0}^{r}$, there are unique $V_{1} \in M_{0}^{1}$ and $V_{2} \in Z_{0}^{r}$ such that $V=V_{1}+V_{2}$, and for $g$ defined on each $I_{i}$ and $g \in L^{2}(I)$, let

$$
I(g, V)=D\left(g, V_{2}\right)+\int_{0}^{1} g(x) V_{1}(x) d x .
$$

For $g \in H^{1}(I)$ such that $g^{\prime \prime}$ is defined on each $I_{i}$,

$$
D(g, V)=-D\left(g^{\prime \prime}, V_{2}\right)+\int_{0}^{1} g^{\prime}(x) V_{1}^{\prime}(x) d x .
$$

If $g, V \in M_{0}^{r}$, relationship (2) and integration by parts imply that

$$
D(g, V)=\int_{0}^{1} g^{\prime}(x) V^{\prime}(x) d x .
$$

Notice that if $p \in \mathbf{P}_{2 r-2}(0,1)$, then $p(x)=p_{2 r-2} \cdot J_{r-1}^{2}(x)+q(x)$, where $p_{2 r-2}$ is the coefficient of $x^{2 r-2}$ in $p(x)$, and $q \in \mathbf{P}_{2 r-3}(0,1)$. Then it is straightforward to show that

$$
\begin{aligned}
\sum_{j=1}^{r-1} \omega_{j} p\left(\varphi_{j}\right)= & \int_{0}^{1} x(1-x) p(x) d x \\
& +\frac{1}{(2 r) !} \frac{d^{2 r}}{d x^{2 r}}[x(1-x) p(x)] \int_{0}^{1} x(1-x) J_{r-1}^{2}(x) d x .
\end{aligned}
$$

From this relationship it follows that if $g \in M_{0}^{r}$, then

$$
I(g, g) \geqslant \int_{0}^{1} g^{2}(x) d x .
$$

When considering functions of more than one variable a subscript, $x$ or $y$, will be used to denote the variable to which the bilinear form is being applied. Bilinear forms on the two variables are formed by taking tensor products of $D$ and $I$. From (4), it follows that

$$
M=\left(Z_{0}^{r} \otimes Z_{0}^{r}\right) \oplus\left(Z_{0}^{r} \otimes M_{0}^{1}\right) \oplus\left(M_{0}^{1} \otimes Z_{0}^{r}\right) \oplus\left(M_{0}^{1} \otimes M_{0}^{1}\right) \equiv N_{1} \oplus N_{2} \oplus N_{3} \oplus N_{4} .
$$

Thus, for $V \in M$ there are unique $V_{m} \in N_{m}, m=1, \ldots, 4$, such that

$$
V=V_{1}+V_{2}+V_{3}+V_{4} \text {. }
$$

And for $g$ an $L^{2}$-function defined on all of $\Omega$, let

$$
\begin{aligned}
I_{x} \otimes I_{y}(g, V)= & \sum_{i, l=0}^{N}\left\{\sum_{k=1}^{r-1} \sum_{j=1}^{r-1} h_{i} h_{l} \omega_{k} \omega_{j} \frac{g\left(x_{i j}, y_{l k}\right) V_{1}\left(x_{i j}, y_{l k}\right)}{\varphi_{k}\left(1-\varphi_{k}\right) \varphi_{j}\left(1-\varphi_{j}\right)}\right\} \\
& +\int_{I} D\left(g(\cdot, y), V_{2}(\cdot, y)\right) d y+\int_{I} D\left(g(x, \cdot), V_{3}(x, \cdot)\right) d x+\left(g, V_{4}\right)
\end{aligned}
$$


The other bilinear forms $D_{x} \otimes I_{y}$ and $I_{x} \otimes D_{y}$ are similarly defined. Using these bilinear forms, a related variational approximation is defined. Let $W \in M$ be the solution to

$$
D_{x} \otimes I_{y}(W, V)+I_{x} \otimes D_{y}(W, V)=I_{x} \otimes I_{y}(f, V), \quad \forall V \in M .
$$

It follows from the definition of the bilinear forms that $U \in M$, the solution to (3), satisfies equations (7). Thus, in order to show existence and uniqueness of $U \in M$ satisfying (3) it is enough to show that if $f=0$ then $W=0$. Relationship (5) implies that $W$ satisfies

$$
\int_{0}^{1} I_{y}\left(W_{x}, V_{x}\right) d x+\int_{0}^{1} I_{x}\left(W_{y}, V_{y}\right) d y=I_{x} \otimes I_{y}(f, V), \quad \forall V \in M .
$$

Choosing $V=W$ and using (6), it follows that

$$
\int_{0}^{1} \int_{0}^{1} W_{x}^{2} d x d y+\int_{0}^{1} \int_{0}^{1} W_{y}^{2} d x d y \leqslant I_{x} \otimes I_{y}(f, W)
$$

thus

$$
(\nabla W, \nabla W) \leqslant I_{x} \otimes I_{y}(f, W)
$$

hence if $f=0, W=0$.

Error Analysis. As before let $u$ denote the solution to the boundary value problem (1) and $U \in M$ the collocation-Galerkin solution to (7). In this section, estimates for $u-U$ are derived. Those estimates are given in the following theorem.

THEOREM. Let $u$ be the solution to (1) and $U \in M$ the collocation-Galerkin approximation to $u$ defined by (3). Then, if $u$ is sufficiently smooth, there exists a constant $C$ independent of $h$ and $u$ such that

$$
\|u-U\|_{L^{2}}+h\|u-U\|_{H_{0}^{1}} \leqslant C h^{s}\|u\|_{H^{s}}, \quad 1 \leqslant s \dot{\leqslant}+1 .
$$

Before proving the theorem, some basic estimates are derived. For $e=u-U$, let $\psi \in H^{2}(\Omega)$ satisfy

then

$$
\begin{aligned}
-\Delta \psi=e, & \text { on } \Omega, \\
\psi=0, & \text { on } \partial \Omega,
\end{aligned}
$$

$$
\|e\|_{L^{2}}^{2}=(e, e)=-(e, \Delta \psi)=(\nabla e, \nabla \psi)=(\nabla e, \nabla(\psi-\chi)), \quad \chi \in M_{0}^{1} \otimes M_{0}^{1},
$$

using (3.iv). Thus, by Cauchy-Schwarz inequality

$$
\|e\|_{L^{2}}^{2} \leqslant\|e\|_{H_{0}^{1}}\|\psi-\chi\|_{H_{0}^{1}}, \quad \chi \in M_{0}^{1} \otimes M_{0}^{1},
$$

the approximation properties of the space $M_{0}^{1} \otimes M_{0}^{1}$ imply that

$$
\inf _{\chi \in M_{0}^{1} \otimes M_{0}^{1}}\|\psi-\chi\|_{H_{0}^{1}} \leqslant C h\|\psi\|_{H^{2}},
$$

also by elliptic regularity

$$
\|\psi\|_{H^{2}} \leqslant C\|e\|_{L^{2}},
$$

thus it follows from these inequalities that 


$$
\|e\|_{L^{2}} \leqslant C h\|e\|_{H_{0}^{1}} .
$$

Therefore, it is sufficient to derive an $H_{0}^{1}$-norm estimate of the error. In the derivation of this estimate the following lemma, proved in [3], will play an important role.

Lemma 1. $\Phi \in H^{r-1}(0,1)$, let $g \in H^{2}(0,1) \cap H_{0}^{1}(0,1)$ satisfy $g^{\prime \prime}=\Phi$. Also, let $G \in M_{0}^{r}$ be the collocation-Galerkin approximation to $g$ satisfying

and

$$
G^{\prime \prime}\left(x_{i j}\right)=\Phi\left(x_{i j}\right), \quad i=0, \ldots, N ; j=1, \ldots, r-1,
$$

$$
\int_{0}^{1} G^{\prime} V^{\prime} d x+\int_{0}^{1} \Phi V d x=0, \quad \forall V \in M_{0}^{1}
$$

then, there exists a constant $C$ independent of $h$ such that

$$
\int_{0}^{1}|\epsilon|^{2} d x+h^{2} \int_{0}^{1}\left|\epsilon^{\prime}\right|^{2} d x \leqslant C h^{2 s} \int_{0}^{1}\left|\frac{d^{s}}{d x^{s}} g\right|^{2} d x, \quad 1 \leqslant s \leqslant r+1,
$$

where $\epsilon=g-G$.

Also, a space $M_{x}$ and an element $q \in M_{x}$, a projection of $u$ into $M_{x}$, are introduced. Then error estimates for $u-q$ and $q-U$ will be obtained in Lemmas 2 and 3 , respectively. The desired estimates will follow from the triangle inequality.

The space $M_{x}$ is defined by

$$
M_{x}=\left\{\sum_{s} \alpha_{s}(y) V_{s}(x),\left\{V_{s}\right\} \text { basis for } M_{0}^{r} \text { and } \alpha_{s} \in H_{0}^{1}(I) \cap H^{2}(I)\right\} .
$$

The function $q \in M_{x}$ satisfies the following equations

$$
\begin{gathered}
(u-q)_{x x}\left(x_{i j}, y\right)+(u-q)_{y y}\left(x_{i j}, y\right)=0, \\
i=0, \ldots, N ; j=1, \ldots, r-1, y \in(0,1), \\
-\int_{0}^{1}(u-q)_{x}(\xi, y) V^{\prime}(\xi) d \xi+\int_{0}^{1}(u-q)_{y y}(\xi, y) V(\xi) d \xi=0, \\
V \in M_{0}^{1}, y \in(0,1) .
\end{gathered}
$$

This system of equations leads to a system of second order two-point boundary value problems, the collocation-Galerkin solution of which corresponds to the solution $U$ of (3). Moreover, $U$ satisfies

$$
D_{x} \otimes I_{y}(U-q, V)+I_{x} \otimes D_{y}(U-q, V)=0, \quad \forall V \in M .
$$

This fact will play an important role later. Using the bilinear forms $D$ and $I$, a weak formulation of (10) can be written as

$$
\int_{0}^{1} D_{x}((u-q), v) d y+\int_{0}^{1} I_{x}\left((u-q)_{y}, v_{y}\right) d y=0, \quad \forall v \in M_{x} .
$$

The choice $v=q$ and the use of relationships (5) and (6) in (12) give

$$
\int_{0}^{1} \int_{0}^{1} q_{x}^{2} d x d y+\int_{0}^{1} \int_{0}^{1} q_{y}^{2} d x d y \leqslant \int_{0}^{1} D_{x}(u, q) d y+\int_{0}^{1} I_{x}\left(u_{y}, q_{y}\right) d y
$$

from which existence and uniqueness of $q$ follow. Notice that if for any $\alpha \geqslant 0$, $\partial^{\alpha} u / \partial y^{\alpha} \in H=H_{0}^{1}(\Omega) \cap H^{2}(\Omega)$, then $\partial^{\alpha} q / \partial y^{\alpha} \in M_{x}$. The estimates of $u-q$ are giv. en in the following 1 :mma. 
Lemma 2. Let $u$ be the solution to (1) and $q \in M_{x}$ the solution to (12). Then, if $u$ is sufficiently smooth, there exists a constant $C$ independent of $h$ such that, for $\alpha \geqslant 0$

$$
\begin{aligned}
\| \frac{\partial^{\alpha}}{\partial y^{\alpha}}(u-q) & \left\|_{L^{2}}+h\right\| \frac{\partial^{\alpha+1}}{\partial x \partial y^{\alpha}}(u-q) \|_{L^{2}} \\
& \leqslant C h^{s}\left\{\left\|\frac{\partial^{s+\alpha} u}{\partial x^{s} \partial y^{\alpha}}\right\|_{L^{2}}+\left\|\frac{\partial^{s+\alpha} u}{\partial x^{s-1} \partial y^{\alpha+1}}\right\|_{L^{2}}\right\}, \quad 1 \leqslant s \leqslant r+1 .
\end{aligned}
$$

Proof. Let $\eta=u-q$. It is straightforward to show that equations (10.ii) imply that

$$
\|\eta\|_{L^{2}} \leqslant C h\left\|\nabla \eta_{x}\right\|_{L} 2 .
$$

Thus, it suffices to estimate $\left\|\eta_{x}\right\|_{L^{2}}$. For this a map $w \in M_{x}$, for which the results of Lemma 1 can be applied directly, is introduced. Estimates of the difference of $w$ and $q$ will be derived and estimates for $u-q$ will follow from the triangle inequality. Define the map $w$ by

$$
(u-w)_{x x}\left(x_{i j}, y\right)=0, \quad i=0, \ldots, N ; j=1, \ldots, r-1 ; y \in(0,1)
$$

and

$$
\int_{0}^{1}(u-w)_{x}(\xi, y) V_{x}(\xi) d \xi=0, \quad V \in M_{0}^{1}, y \in(0,1)
$$

Equivalently, $w$ is the solution of

$$
\int_{0}^{1} D_{x}(u-w, v) d y=0, \quad v \in M_{x} .
$$

Notice that, as for $q$, if for any $\alpha \geqslant 0, \partial^{\alpha} u / \partial y^{\alpha} \in H$, then $\partial^{\alpha} w / \partial y^{\alpha} \in M_{x}$. From Lemma 1 it follows that there exists a constant $C$ independent of $h$ satisfying for $\alpha \geqslant 0$,

$$
\left\|\frac{\partial^{\alpha}(u-w)}{\partial y^{\alpha}}\right\|_{L^{2}}+h\left\|\frac{\partial^{\alpha+1}}{\partial x \partial y^{\alpha}}(u-w)\right\|_{L^{2}} \leqslant C h^{s}\left\|\frac{\partial^{s+\alpha}}{\partial x^{s} \partial y^{\alpha}} u\right\|_{L^{2}}, \quad 1 \leqslant s \leqslant r+1 .
$$

Estimates of the difference between $w$ and $q$ are now derived. Let $\epsilon=q-w$. Equations (12) and (15) imply

$$
\int_{0}^{1} D_{x}(\epsilon, v) d y+\int_{0}^{1} I_{x}\left((\epsilon)_{y}, v_{y}\right) d y=\int_{0}^{1} I_{x}\left((u-w)_{y}, v_{y}\right) d y, \quad \forall v \in M_{x} .
$$

Since $\epsilon$ and $v \in M_{x}$, then by (5)

$$
\int_{0}^{1} D_{x}(\epsilon, v) d y=\int_{0}^{1} \int_{0}^{1} \epsilon_{x} v_{x} d x d y=\left(\epsilon_{x}, V_{x}\right),
$$

and for $v=\epsilon$ by (6)

$$
\int_{0}^{1} I_{x}\left(\epsilon_{y}, \epsilon_{y}\right) d y \geqslant \int_{0}^{1} \int_{0}^{1} \epsilon_{y}^{2} d x d y=\left(\epsilon_{y}, \epsilon_{y}\right) .
$$

In [3], it was shown that if $g \in H^{s}(I), 1 \leqslant s \leqslant r+1$ and $V \in M_{0}^{r}$, then

$$
\left|I(g, V)-\int_{0}^{1} g V d x\right| \leqslant C h^{s}\left\{\int_{0}^{1}\left(\frac{d^{s} g}{d x^{s}}\right)^{2} d x\right\}^{1 / 2}\left\{\int_{0}^{1} V^{2} d x\right\}^{1 / 2} .
$$


Thus

$$
\begin{aligned}
\left\|\epsilon_{x}\right\|_{L^{2}}^{2}+\left\|\epsilon_{y}\right\|_{L^{2}}^{2} & \leqslant\left((u-w)_{y}, \epsilon_{y}\right)+\int_{0}^{1}\left\{I_{x}\left((u-w)_{y}, \epsilon_{y}\right)-\int_{0}^{1}(u-w)_{y} \epsilon_{y} d x\right\} d y \\
& \leqslant\left((u-w)_{y}, \epsilon_{y}\right)+\int_{0}^{1}\left\{C h\left(\int_{0}^{1}(u-w)_{x y}^{2} d x\right)^{1 / 2}\left(\int_{0}^{1} \epsilon_{y}^{2} d x\right)^{1 / 2}\right\} d y \\
& \leqslant \frac{1}{4 \epsilon}\left\|(u-w)_{y}\right\|_{L^{2}}^{2}+2 \xi\left\|_{y}\right\|_{L^{2}}^{2}+\frac{C^{2} h^{2}}{4 \xi}\left\|(u-w)_{x y}\right\|_{L^{2}}^{2},
\end{aligned}
$$

where the inequality $2 a b \leqslant a^{2}+b^{2}$ has been used. Take $\xi=1 / 2$, then

$$
\left\|(w-q)_{x}\right\|_{L^{2}}^{2} \leqslant C\left\{\left\|(u-w)_{y}\right\|_{L^{2}}^{2}+h^{2}\left\|(u-w)_{x y}\right\|_{L^{2}}^{2}\right\} .
$$

Estimates (14), (16) and (18) and the triangle inequality complete the proof of the lemma.

It remains to obtain estimates of the difference between $U$ and $q$. In order to obtain these estimates, an auxiliary function $W \in M$ is introduced which satisfies

$$
(W-q)_{y y}\left(x, y_{l k}\right)=0, \quad l=0, \ldots, N ; k=1, \ldots, r-1, x \in(0,1),
$$

$$
-\int_{0}^{1}(W-q)_{y}(x, \xi) V_{y}(\xi) d \xi=0, \quad V \in M_{0}^{1}, x \in(0,1),
$$

or equivalently

In particular

$$
D_{y}(W-q, V)=0, \quad \forall V \in M_{0}^{r}, x \in(0,1)
$$

$$
I_{x} \otimes D_{y}(W-q, V)=0, \quad \forall V \in M .
$$

Equations (19) and Lemma 1 imply that for $\alpha=0,1$

$$
\left\|\frac{\partial^{\alpha}}{\partial x^{\alpha}}(q-W)\right\|_{L^{2}}+h\left\|\frac{\partial^{\alpha+1}}{\partial x^{\alpha} \partial y}(q-W)\right\|_{L^{2}} \leqslant C h^{s}\left\|\frac{\partial^{s+\alpha}}{\partial x^{\alpha} \partial y^{s}} q\right\|_{L^{2}} .
$$

Setting $q=q-u+u$ and using estimate (13), it follows that

(21) $\|q-W\|_{L^{2}}+h\left\{\left\|(q-W)_{x}\right\|_{L^{2}}+\left\|(q-W)_{y}\right\|_{L^{2}}\right\}+h^{2}\left\|(q-W)_{x y}\right\|_{L^{2}} \leqslant C h^{s}\|u\|_{H^{s}}$.

In the following lemma, estimates of the difference between $U$ and $W$ are given.

Lemma 3. Let $U \in M$ be the solution to (3), $q \in M_{x}$ the solution to (12) and $W \in M$ the solution to (19). Then there exists a constant $C$ independent of $h$ such that

$$
\|\nabla(U-W)\|_{L^{2}}^{2} \leqslant C\left\{\left\|(q-W)_{x}\right\|_{L^{2}}^{2}+h^{2}\left\|(q-W)_{x y}\right\|_{L^{2}}^{2}\right\}
$$

Proof. Let $E=U-W$. Using (11) and (20); it follows that

$$
D_{x} \otimes I_{y}(E, V)+I_{x} \otimes D_{y}(E, V)=D_{x} \otimes I_{y}(q-W, V), \quad \forall V \in M,
$$

or equivalently using (5)

$$
\int_{0}^{1} I_{y}\left(E_{x}, V_{x}\right) d x+\int_{0}^{1} I_{x}\left(E_{y}, V_{y}\right) d y=\int_{0}^{1} I_{y}\left((q-W)_{x}, V_{x}\right) d x, \quad \forall V \in M,
$$


with $V=E$ and using (6), it follows that

$$
\left\|E_{x}\right\|_{L^{2}}^{2} \leqslant \int_{0}^{1} I_{y}\left(E_{x}, E_{x}\right) d x
$$

and

$$
\left\|E_{y}\right\|_{L^{2}}^{2} \leqslant \int_{0}^{1} I_{x}\left(E_{y}, E_{y}\right) d y
$$

Hence

$$
\begin{aligned}
\left\|E_{x}\right\|_{L^{2}}^{2}+\left\|E_{y}\right\|_{L^{2}}^{2} & \leqslant \int_{0}^{1} I_{y}\left((q-W)_{x}, E_{x}\right) d y \\
& \leqslant\left((q-W)_{x}, E_{x}\right)+\int_{0}^{1}\left\{I_{y}\left((q-W)_{x}, E_{x}\right)-\int_{0}^{1}(q-W)_{x} E_{x} d x\right\} d y \\
& \leqslant\left((q-W)_{x}, E_{x}\right)+\int_{0}^{1}\left\{C h\left(\int_{0}^{1}(q-W)_{x y}^{2} d y\right)^{1 / 2}\left(\int_{0}^{1} E_{x}^{2} d y\right)^{1 / 2}\right\} d x \\
& \leqslant \frac{1}{4 \epsilon}\left\|(q-W)_{x}\right\|_{L^{2}}^{2}+2 \epsilon\left\|E_{x}\right\|_{L^{2}}^{2}+\frac{C^{2} h^{2}}{4 \epsilon}\left\|(q-W)_{x y}\right\|_{L^{2}}^{2},
\end{aligned}
$$

where the inequality $2 a b \leqslant a^{2}+b^{2}$ has been used. With $\epsilon=1 / 4$ and $\epsilon=1 / 2$, estimate (22) follows completing the proof of the lemma.

The Theorem follows from estimates (9), (13), (21) and (22) and the triangle inequality.

Remarks. 1. Although the same partition has been used for both sides, the argument holds for different partitions.

2. The Galerkin procedure for the same space requires $\{(N+1)(r-1)+N\}^{2}$ two-dimensional quadratures while the procedure described by (3) needs only $N^{2}$ twodimensional quadratures plus $2(N+1)(r-1)$ one-dimensional quadratures.

3. In general the solution can only be asserted to lie in $H^{3-\epsilon}(\Omega)$, for any $\epsilon>0$.

Department of Mathematics

University of Kentucky

Lexington, Kentucky 40506

1. D. ARCHER \& J. C. DIAAZ, "A collocation-Galerkin method for a first order hyperbolic equation." (To appear.)

2. JULIO CÉSAR DIAZ VELASCO, A Hybrid Collocation-Galerkin Method for the Two Point Boundary Value Problem Using Continuous Piecewise Polynomials Spaces, Ph.D. Thesis, Rice Univ., Houston, Texas, 1974.

3. J. C. DÍAZ, "A collocation-Galerkin method for the two point boundary value problem using continuous piecewise polynomial spaces," SIAM J. Numer. Anal., v. 14, 1977, pp. 844-858.

4. R. J. DUNN \& M. F. WHEELER, "Some collocation-Galerkin methods for two-point boundary-value problems," SIAM J. Numer. Anal., v. 13, 1976, pp. 720-733.

5. P. M. PRENTER \& R. D. RUSSELL, "Orthogonal collocation for elliptic partial differential equations," SIAM J. Numer. Anal., v. 13, 1976, pp. 923-939.

6. M. F. WHEELER, "A $C^{0}$ collocation-Galerkin method for two-point boundary-value problems and one space dimensional parabolic problems," SIAM J. Numer. Anal., v. 14, 1977, pp. $71-90$. 